\title{
Fluoride dentifrice overcomes the lower resistance of fluorotic enamel to demineralization
}

\author{
Almeida LF ${ }^{1}$, Marín LM ${ }^{1}$, Martínez-Mier EA ${ }^{2}$, Cury JA ${ }^{1}$ \\ ${ }^{1}$ Piracicaba Dental School, University of Campinas, Piracicaba, SP, Brazil \\ ${ }^{2}$ Indiana University School of Dentistry, Department of Cariology, Operative Dentistry \\ and Dental Public Health, Indianapolis, Indiana, USA
}

Short Title: F-dentifrice makes fluorotic enamel more resistant to demineralization

\section{Corresponding author:}

Prof. Jaime Aparecido Cury

Piracicaba Dental School, UNICAMP

CP 52

13414-903, Piracicaba, SP, Brazil

Phone: $+55-19-2106-5303$

email: jcury@unicamp.br

Key words: Dental caries, Dental fluorosis, Dentifrice, Fluoride, Hypomineralization,

\section{DISCLOSURE STATEMENT}

The authors have no conflicts of interest to declare. 


\section{ABSTRACT}

We evaluated if the low resistance of fluorotic enamel to demineralization could be overcome by the fluoride dentifrice (FD) treatment. Paired enamel slabs of sound and fluorotic enamel ( $n=20 /$ group) from human teeth presenting TF fluorosis index from 0 to 4 were obtained. Half of the anatomic surface of enamel slabs was isolated and used as a control (baseline) of enamel mineralization and fluoride concentration. The slabs were submitted to a $\mathrm{pH}$-cycling model simulating a high cariogenic challenge and $2 x /$ day they were treated with placebo dentifrice (PD) or FD (1,100 $\mu \mathrm{g} \mathrm{F/g,} \mathrm{as} \mathrm{NaF).} \mathrm{After} 10$ days, the slabs were cut in two halves. Enamel demineralization was evaluated by cross sectional microhardness in one half, and the fluoride formed (FF) concentration was determined in the other half. For statistical analysis, the data of net demineralization area $(\Delta \Delta \mathrm{S})$ and FF ( $\mu \mathrm{g} \mathrm{F/g}$ ) were grouped as follows: TF0, TF1-2, and TF3-4, and analyzed by two-way ANOVA followed by Tukey test $(\alpha=5 \%)$. The factors under study were TF $(0$, 1-2 and 3-4) and dentifrice treatment (PD or FD). The effect of the factors was statistically significant for $\Delta \Delta S$ and FF ( $p<0.05)$. In PD group, $\Delta \Delta S$ was TF3-4>TF1-2>TF0 $(p<0.05)$, but the groups did not differ ( $p>0.05$ ) when FD was used. For FF, the groups treated with PD did not differ $(p>0.05)$ but greatest $(p<0.05)$ FF concentration was found in group TF3-4 treated with FD. These findings suggest that the higher susceptibility of fluorotic enamel to demineralization lesions is decreased by the use of fluoridated dentifrice. 


\section{INTRODUCTION}

Irrespective of the caries decline reported worldwide [Petersen et al., 2005; Do, 2012], the anticaries benefits of water fluoridation continue to be observed even in developed countries, such as Australia [Spencer et al., 2018], Ireland [Mullen et al., 2012] and the United States [Slade et al., 2018]. Since the 1950s, the benefits and risks of fluoridated water use have been debated worldwide [Burt, 1992; Spencer et al., 2018]. Water fluoridation is considered an acceptable community-based method for fluoride delivery, because the risk of developing dental fluorosis lesions by the ingestion of fluoride during the enamel formation period has been deemed acceptable when contrasted to fluoride's anticaries benefits [Petersen and Lennon, 2004]. Very mild and mild fluorosis lesions resulting from optimally-fluoridated water consumption do not appear to result in aesthetic concerns [Riordan, 1993] or affect people's quality of life [Chankanka et al., 2010].

In the past, it was considered that the systemically ingested fluoride would exert its primary preventive effect after being incorporated into the enamel as fluorapatite, making the enamel more resistant to the caries process [Fejerskov et al., 1981]; however, it is now recognized that the main effect of water fluoridation is local and post eruptive [ten Cate, 1999]. At the compositional level, fluorotic enamel presents greater fluoride concentration than sound enamel [Richards at al., 1989]. In addition to its higher fluoride content, fluorotic enamel is characterized for being hypomineralized [Thylstrup \& Fejerskov, 1978] and it has been proposed that this higher porosity could make it more susceptible to caries.

The hypothesis whether fluorotic enamel is more resistant or not to the development of carious lesions has been experimentally tested for a long time with contradictory results. While some authors found no difference in the severity of the lesions created on fluorotic or non-fluorotic enamel [Alhawij et al., 2015], others have reported moderately fluorotic enamel either to be more susceptible [Suma et al. 2008] or more resistant to demineralization [Kidd et al., 1978; Kidd et al., 1980; Waidyasekera et al., 2007] than non-fluorotic enamel. According to Marin et al., [2016], the lack of agreement among these in vitro studies may be explained by differences in sample preparation methods used to induce caries lesions' formation or the methods used to compare the change of mineral content between sound and fluorotic teeth.

Using a validated pH-cycling model [Argenta et al.,2003] to overcome the possible experimental gaps of previous studies, Marin et al., [2016] showed that the 
enamel with higher fluorosis severity (TF3-4) was less resistant to demineralization than sound enamel (TF0), even though the fluoride concentration found in fluorotic enamel was significantly higher than the sound enamel in the study. According to the authors, the higher porosity of the enamel in TF3-4 teeth could be the reason for the increased demineralization found because: i) acid diffusion into enamel could be facilitated, and ii) the higher porosity results in a greater mineral area to be dissolved by the acids [Marin et al., 2016]. However, the caries process induced by the $\mathrm{pH}$-cycling regimen used by Marin et al., [2016] was achieved in total absence of the local effect of fluoride.

Therefore, we hypothesized that the use of fluoride dentifrice could overcome the lower resistance of fluorotic enamel to demineralization and conducted the present study to add to the findings of our previous study [Marin et al., 2016].

\section{MATERIALS AND METHODS}

\section{Experimental Design}

This study was approved by the Piracicaba Dental School (UNICAMP) Research and Ethics Committee (protocol: 1.348.963).

An in vitro study with factorial design was conducted. The factors were: fluorosis at three severity levels (TF 0,1-2, 3-4), classified according to the Thylstrup and Fejerskov index [TF, Thylstrup \& Fejersvok, 1978]; and dentifrice at two levels: Fluoride dentifrice (FD, $1100 \mu \mathrm{g} \mathrm{F} / \mathrm{g}$ ) and placebo (PD, without fluoride). Twenty unerupted third molars without dental fluorosis (TF0) and 80 with dental fluorosis (TF1-4) were selected for this study. Two enamel slabs $(4 \times 3 \times 2 \mathrm{~mm})$ were obtained from each tooth, and each one was allocated into each dentifrice groups $(n=20)$. All slabs had half of the anatomic surface isolated with nail varnish to avoid the contact with the de- and remineralizing solutions, as well as with the dentifrice treatments. This non-exposed area was used as a control to measure hypomineralization and fluoride concentration (baseline data). The slabs were subjected to a pH-cycling model and treated with PD or FD 2x/day. After 10 days, the slabs were cut, and the enamel demineralization was evaluated by cross sectional microhardness in one half, while the other half was used to assess the fluoride formed (FF) concentration after acid etching, determined with a fluoride electrode (Fig.1). For statistical analysis, net demineralization area $(\Delta \Delta \mathrm{S})$ and FF ( $\mu \mathrm{g} \mathrm{F/g})$ calculated for TF 0 , TF1-2, and TF3-4 were analyzed by two-way ANOVA followed by Tukey's test ( $\alpha=5 \%)$. 
Unerupted third molars, extracted for clinical reasons, were obtained from the Teeth Banks of the University of São Paulo (Brazil; sound teeth) and the Indiana University School of Dentistry (United States; fluorotic teeth) and stored in a $0.2 \%$ thymol solution at $4{ }^{\circ} \mathrm{C}$. Twenty sound teeth (TF0) and 80 with fluorosis (TF1-4, $\mathrm{n}=20 /$ each TF group) were selected by two previously trained examiners. Only teeth with TF scores up to 4 were used because unerupted teeth do not present higher TF scores [Baelum et. al.,1986]. Teeth which presented other developmental or mechanical defects were excluded. To obtain the enamel slabs, the teeth were cut in the coronal third where the enamel surface is flat. Two enamel slabs $(4 \times 3 \times 2 \mathrm{~mm})$ were obtained from each tooth. All surfaces of each slab were covered with nail varnish, except for only half of the anatomic surface, with an area of $6 \mathrm{~mm}^{2}$, exposed to the $\mathrm{pH}$-cycling model and treatments. The isolated area was used as a control (baseline) of the hypomineralization and fluoride concentration, and to normalize the data. Each slab was fixed with wax to stainless steel holders to facilitate the immersion in the solutions during the $\mathrm{pH}$-cycling regimen and the treatments with the dentifrices.

\section{pH-cycling regimen}

The $\mathrm{pH}$-cycling model used [Argenta et al., 2003] was previously validated in terms of dose response to evaluate fluoride dentifrice concentration effects on the process of caries lesions development. It produces caries lesions with a relatively well-preserved surface layer. This model was modified by Marín et al. [2016] to differentiate the hypomineralization of fluorotic teeth from the demineralization caused by a $\mathrm{pH}$-cycling regimen. In each cycle, the enamel slabs were first treated with an aqueous slurry of PD or FD (1:4) for 5 min and then washed with purified water, dried in absorbed paper and kept immersed in demineralizing solution $\left(6.37 \mathrm{~mL} / \mathrm{mm}^{2}\right.$ of exposed enamel) for $6 \mathrm{~h}$. After the demineralizing period, the blocks were again treated with a slurry of PD or FD for 5 min, washed, dried, and then immersed in remineralizing solution $\left(3.18 \mathrm{~mL} / \mathrm{mm}^{2}\right)$ for 18 $\mathrm{h}$. The experiment was composed of 10 cycles, and before starting the $6^{\text {th }}$ cycle, the solutions were changed to maintain their saturation degree with respect to the enamel. The demineralizing solution was unsaturated with respect to hydroxyapatite and fluorapatite and was composed of $2.0 \mathrm{mM}$ calcium, $2.0 \mathrm{mM}$ phosphate, and $0.03 \mu \mathrm{g} \mathrm{F} / \mathrm{ml}$, in $75 \mathrm{mM}$ acetate buffer, $\mathrm{pH} \mathrm{4.3.} \mathrm{The} \mathrm{remineralizing} \mathrm{solution} \mathrm{was} \mathrm{supersaturated} \mathrm{with}$ respect to hydroxyapatite and fluorapatite and was composed of $1.5 \mathrm{mM}$ calcium, 0.9 $\mathrm{mM}$ phosphate, $150 \mathrm{mM} \mathrm{KCl}$, and $0.05 \mu \mathrm{g} \mathrm{F} / \mathrm{ml}$ in $20 \mathrm{mM}$ cacodylate buffer, $\mathrm{pH}$ 7.4. After 10 cycles, the slabs were collected and stored at $4^{\circ} \mathrm{C}$ under $100 \%$ humidity until analysis. 
Determination of net demineralization area $(\Delta \Delta \mathrm{S})$

Microhardness was used as the indicator of demineralization because there is a high correlation between enamel cross sectional microhardness (CSMH) and the percentage of mineral volume $(\% \mathrm{vol})$ determined by transverse microradiography (TMR) [Featherstone et al., 1983; Kielbassa et al., 1999], as with incipient caries lesions [Cury et al., 2000].

For $\Delta \Delta \mathrm{S}$ determination, the slabs $(4 \times 3 \times 2 \mathrm{~mm})$ were longitudinally cut to obtain two hemi slabs $(4 \times 1.5 \times 2 \mathrm{~mm})$, having half of the exposed and non-exposed areas each (Fig 1$)$. One hemi slab was embedded in acrylic resin, and the cut surface was flattened and polished. CSMH analysis was performed using a microhardness tester [Future-Tec FM Corp, Tokyo, Japan] coupled to the FM-ARS analysis software, using a Knoop indenter with a 25-gram load for $5 \mathrm{~s}$. In the exposed and non-exposed enamel regions, three rows of 10 indentations were made in the central region separated $100 \mu \mathrm{m}$ from each other. The indentations were made from the outer enamel surface at $10 \mu \mathrm{m}$ up to $400 \mu \mathrm{m}$. The mean values at all measuring points at each distance were then averaged. The hypomineralization $\left(\mathrm{S}_{\text {hypo }}\right)$ and the demineralization post-pH cycling $\left(\mathrm{S}_{\text {post-pH-cycling }}\right)$ areas were calculated by the numerical integration of the hardness versus depth values $\left(\mathrm{kg} / \mathrm{mm}^{2} \times \mu \mathrm{m}\right)$, using the trapezoidal rule [Cury et al., 2010]. The hypomineralization

$\left(\Delta S_{\text {hypo }}=S_{\text {sound }}-S_{\text {fluorotic }}\right)$ and induced demineralization $\left(\Delta S_{\text {post-pH-cycling }}=S_{\text {sound }}-S_{\text {post-pH }}\right.$ cycling) areas were calculated. Finally, the $\Delta \Delta \mathrm{S}$ was obtained $\left(\Delta \Delta \mathrm{S}=\Delta \mathrm{S}\right.$ post-pH-cycling $\left.-\Delta \mathrm{S}_{\text {hypo }}\right)$ which represents the increase of integrated area of hypomineralization $(\Delta S)$ during the $\mathrm{pH}$-cycling under the effect of the treatments with the dentifrices.

\section{Determination of Fluoride Formed (FF) on enamel}

The remaining hemi slab $(4 \times 1.5 \times 2 \mathrm{~mm})$ was used for FF analysis (Fig 1). It was cut to separate the post $\mathrm{pH}$-cycling enamel (exposed) from the enamel non-exposed to the $\mathrm{pH}$ cycling regime. The two quarters $(2 \times 1.5 \times 2)$ of slab had all surfaces, except the anatomic surface protected with wax and they were subjected to acid etch for fluoride analysis [Marin et al., 2016]. The enamel surface of each slab was successively etched with volumes of $250 \mu \mathrm{L}$ of $0.5 \mathrm{M} \mathrm{HCl}$ for $15,30,60$, and $120 \mathrm{~s}$ under agitation at $150 \mathrm{rpm}$ to remove four enamel layers. Each acid extract was buffered with $250 \mu \mathrm{L}$ of TISAB II containing $20 \mathrm{~g} \mathrm{NaOH} / \mathrm{L}$. Fluoride and $P_{\mathrm{i}}$ were determined in each extract as described by Marin et al., [2016]. The amount (g) of enamel dissolved was calculated based on the enamel \%Pi found for each TF [Marin et al., 2016], allowing the calculation of the fluoride 
concentration ( $\mu \mathrm{g} \mathrm{F/g}$ ) in each layer removed. The layer $(\mu \mathrm{m})$ of enamel removed was estimated based on the amount found of enamel removed and fixing the density in 2.92 $\mathrm{g} / \mathrm{ml}$ [Cury et al., 2000]. The data were expressed as fluoride concentration ( $\mu \mathrm{g} \mathrm{F/g}$ ) found at each distance of enamel surface and total fluoride concentration. The total fluoride concentration was obtained summing the amount of fluoride found $(\mu \mathrm{g})$ in the four layers of enamel removed and dividing by the sum of the weights $(\mathrm{g})$ of enamel. For the statistical analysis, the concentration of fluoride found in the exposed half was subtracted from that found in the baseline to obtain the net concentration of fluoride formed (FF). Thus, FF represents the increase of fluoride concentration in enamel due to the treatments.

\section{Statistical Analysis}

In order to increase the power of the statistical analysis, TF 1 and 2, and TF 3 and 4 were combined into two groups. Then, the data obtained from the three resulting groups (TF0, TF1-2 and TF3-4) were statistically analyzed. The assumptions of equality of variances and normal distribution of errors were checked for the response variables. For $\Delta \Delta S$ data, the statistical program highlighted an outlier that was excluded from the data. For $\mathrm{FF}$, the data were transformed to $\log _{10}$. After these required adjustments, all groups presented normal distribution and equality of variances. The data were analyzed by twoway ANOVA, followed by Tukey test. All analyses were performed in the Statistical Package for Social Science [SPSS, IBM- version 20.0] and the significance level was set at $5 \%$.

\section{RESULTS}

Statistical analysis showed significant effects for the factors under study (Fluorosis and Dentifrice) and showed a significant interaction between factors for both variables (Table 1).

The profile of hardness $\left(\mathrm{kg} / \mathrm{mm}^{2}\right)$ throughout the enamel (up to $400 \mu \mathrm{m}$ ) before (baseline) and after the $\mathrm{pH}$-cycling regimen are illustrated in Figure 2. While Figure 2a shows the profile for the enamel treated with placebo dentifrice (PD), Figure $2 b$ highlights data obtained with fluoride dentifrice (FD). Figure $2 a$ shows that the baseline hardness of groups TF0, TF1-2 and TF3-4 is different, with lower values for TF3-4. Figure 2a also shows that the hardness of all groups decreased proportionally to their fluorosis severity after the caries development by the $\mathrm{pH}$-cycling regimen used and that PD treatment was not effective to reduce demineralization. Furthermore, the data on Figure 2a suggest strongly that the demineralization caused in enamel with TF3-4 was greater compared 
to that of the TF1-2 and TF0 groups. On the other hand, Figure $2 b$ suggests that treatment with FD was able to reduce the demineralization caused by $\mathrm{pH}$-cycling; it can also be observed that the lower resistance to demineralization of the enamel with the higher fluorosis severity (TF3-4) was decreased by the use of FD.

The qualitative findings shown in Figures $2 a$ and $2 b$ were confirmed quantitatively by the increase in the integrated area of demineralization caused by $\mathrm{pH}$ cycling $(\Delta \Delta S)$, allowing us to evaluate the effect of the treatments with the dentifrices (Fig. 3). The effect of dentifrice treatment was statistically significant (Table 1) with lower values for FD. Figure 3 shows that in the absence of FD treatment (PD group), the net integrated demineralized area $(\Delta \Delta S)$ was greatest in the fluorotic enamel TF3-4, followed by TF1-2 and TF0 $(p<0.05)$. On the other hand, FD treatment was not only effective to reduce the demineralization in the fluorotic enamel by, but the difference among groups were decreased $(p>0.05)$.

Figure 4 illustrates the profile of fluoride distribution throughout the enamel before (baseline) and after the $\mathrm{pH}$-cycling regimen and treatments with dentifrices (Fig. $4 a$ for $P D$ and $4 b$ for FD). Typical curves were found with higher fluoride concentration at the outermost enamel surface. The effect of the treatments with dentifrices was statistically significant (Table 1) with greater concentration for the groups treated with FD. Figure $4 b$ shows that the effect of FD extends up to the 3rd layer of removed enamel, around $70 \mu \mathrm{m}$ from the dental surface. Also, the data suggest that fluorotic enamel with TF3-4 gained more fluoride than TF1-2 and TF0, mainly in the two outer analyzed layers of enamel. It is noteworthy that this phenomenon found for enamel TF3-4 (subjected to a pH-cycling regimen) and treated with FD (Fig. 4b) is also observed for PD (Fig. 4b).

The qualitative representation of fluoride in enamel showed in Figures $4 a$ and $4 b$ was quantified by the calculation of the net fluoride concentration due to the treatments (see M\&M). Figure 5 shows the concentration of fluoride formed (FF) in enamel due to the treatment with the dentifrices during the $\mathrm{pH}$-cycling regimen. The groups did not differ statistically for the treatment with PD $(p>0.05)$ but higher fluoride concentration was found for TF3-4 treated with FD $(p<0.05)$.

\section{DISCUSSION}

Fluoride affects the initiation and progression of caries because it interferes with the development of carious lesions, reducing the demineralization and enhancing remineralization, which occur when the biofilm accumulates onto the dental surfaces exposed to dietary sugars [Cury et al., 2016]. However, the effectiveness of fluoride to 
arrest or repair early caries lesions is a controversial subject [Cury and Tenuta, 2009]. Similar to early caries lesions, fluorotic enamel presents a porous and hypomineralized subsurface area [Fejerskov et al., 1975]. However, unlike early caries lesions, fluorotic teeth have immature mineralized enamel [Chen and Eisenmann, 1984], whereas that of enamel of carious lesions is restructured due to the caries process [Moreno and Zahradnik, 1974]. Also, the diffusion of fluoride throughout the porosity of fluorotic enamel may be different than the diffusion pattern of this ion across caries lesions. The physicochemical effects of fluoride on the arrestment of early caries lesions is limited [Holmen et al., 1987; Fejerskov and Larsen, 2015] but given the previously described differences, it might be more effective on fluorotic enamel. Therefore, we hypothesized that fluoride from dentifrice treatment could overcome the lower resistance of fluorotic enamel to demineralization.

First, our findings confirmed (Figures $2 a$ and 3 ) that in the absence of FD treatment, fluorotic enamel is in fact less resistant to the caries process than sound enamel. In our previous study [Marin et al., 2016], only the TF3-4 group differed statistically from the TF0, but the present results showed that the group TF1-2 was also less resistant to demineralization than the sound TF0 group (Fig. 3). The current result may be explained by three factors: the origin of the teeth (Colombia-Denmark vs BrazilUSA), the sample size ( $n=20$ vs 40 ), and how the area of demineralization was calculated ( $\Delta S$ vs $\Delta \Delta S$ ). We believe that the sample size is the most important factor to explain our new findings, since the power achieved in the previous study [Marin et al., 2016], using a sample size of 20 , was of 0.70 , while the power achieved in the current study was of 0.99 .

The present results of the demineralization found in the absence of the local effect of fluoride (PD group) reinforces that the fluoride pre-eruptively incorporated to enamel [Fig. 4a-baseline and Marin et al., 2016] is not able to protect fluorotic enamel from increased demineralization $(\Delta \Delta S)$ caused by the caries process induced by a $\mathrm{pH}$-cycling regimen. These findings support to the current concept that the anticaries effect of fluoride is local and post eruptive [Fejerskov et al., 2015]. This finding in agreement with past epidemiological data showing that the incidence of carious lesions increased when children who lived in a fluoridated area moved to one not supplemented with water fluoridation [Russell and Hamilton, 1961] or by the anticaries effect of fluoride in teeth already erupted when a water fluoridation program was implemented [Arnold et al., 1962]. Moreover, our findings (Figs, $2 b$ and 3 ) suggest that children, even those with 
fluorosis, must brush their teeth with fluoride dentifrice to compensate the lower resistance of fluorotic enamel to demineralization.

Indeed, our findings regarding the effect of FD showed that it was not only important to reduce enamel demineralization (Table 1) but also to overcome the lower resistance of fluorotic enamel with TF3-4 to demineralization. Interestingly, the effect of FD reducing the increased demineralization area $(\Delta \Delta S)$ was of the same magnitude for the three groups evaluated, since the enamel of TF3-4 appears to be more susceptible to demineralization in the absence of FD treatment (Fig. 3) did not differ statistically from the enamel presenting TF1-2 and TF0 when treated with FD (Fig. 3).

Although there are data suggesting that caries-like lesions caused in fluorotic enamel are more responsive to fluoride than sound enamel [Alhawij et al., 2015], to the best of our knowledge, the present study is the first one showing that FD is able to prevent further demineralization beyond that already found in hypomineralyzed fluorotic enamel. The effect of fluoride on caries arrestment has been studied for a long time [Yamazaki et al., 2007; Lippert et al., 2012]. For caries, the dose-response effect of fluoride depends directly on the lesion baseline severity $(\Delta Z)$ and lesion mineral distribution [Lippert et al., 2012]. Also, significantly higher concentrations of fluoride (25.0 ppm) were required to prevent further demineralization of artificial caries-like lesions [Yamazaki et al., 2007]. Opposite to those results for caries, our findings showed that FD was able to decrease the lower resistance that fluorotic enamel has to demineralization in comparison to sound enamel (Fig. 3a vs 3b). This result may be explained by the combination of two factors, higher porosity and immature minerals of fluorotic enamel [Fejerskov et al., 1975; Chen and Eisenmann, 1984]. Although the greater porosity may have allowed the acid diffusion to the deepest part of the enamel [Marin et al., 2016], this same pathway is used by fluoride to diffuse into enamel. During the $\mathrm{pH}$-cycling regimen, the period at which the enamel was subjected to the demineralizing solution, the immature enamel containing more soluble salts (carbonate apatite) may have been dissolved while less soluble minerals, as fluoridated apatites, were precipitated [Nelson, 1981; Moreno et al., 1974]. In addition, during the time that the enamel was subjected to the remineralizing solution in the $\mathrm{pH}$-cycling regimen, precipitation of minerals occurs [Fejerskov and Larsen, 2015]. This explanation is supported by our data on fluoride concentration in enamel, as shown in Figures $4 \mathrm{a}$ and $4 \mathrm{~b}$. In addition, as shown in Figure 5 , the higher fluoride concentration found after $\mathrm{pH}$-cycling may be attributed to the effect of the FD treatment because the higher fluoride concentration found in fluorotic enamel at baseline was subtracted. It is noteworthy the coherence between fluoride formed in 
enamel by FD treatment during the $\mathrm{pH}$-cycling regimen (Fig. $4 \mathrm{~b}$ ) and the depth of hypomineralization seen in fluorotic enamel (Fig. 3a). Figure 3a shows that the baseline higher hypomineralization found in the TF3-4 group is seen up to approximately $100 \mu \mathrm{m}$ from enamel surface, while the fluoride concentration found in enamel after the $\mathrm{pH}$ cycling regimen is found around up to $120 \mu \mathrm{m}$ of enamel surface (Fig $4 \mathrm{~b}$ ). Thus, the findings suggest that fluoride was able to diffuse throughout the extension of fluorotic enamel, explaining the efficacy of fluoride arresting further demineralization caused by the caries process induced.

The findings of the present study should not be interpreted as to indicate that toothbrushing with FD is mandatory for people subjected systemically to fluoridated water or salt fluoridation to overcome the low resistance of fluorotic enamel to the caries process, because the local ("topical") effect of these community-based ways of fluoride use was not simulated during the $\mathrm{pH}$-cycling model used here. The local effect of water fluoridation maintaining elevated levels of fluoride in saliva and biofilm [Nobre dos Santos and Cury, 1988] also occurs when foods cooked with water or fluoridated salt are chewed [Lima et al., 2018]. However, up to now there is no model developed to test this local effect. Nevertheless, secondary data of the present study (not presented), showed that the fluoride concentration was higher in the de- and remineralizing solutions where the dental slabs treated with FD were immersed. On average for all groups, fluoride concentrations ( $\mu \mathrm{g} \mathrm{F} / \mathrm{mL}$ ) in the de- and remineralizing solutions of the groups treated with PD were 0.045 and 0.045 , respectively, and for the groups treated with FD were 0.068 and 0.074 . This higher concentrations in the groups treated with FD are expected to occur when water or fluoridated salt are being consumed. In our present study, this higher concentration is due to the dissolution of $\mathrm{CaF}_{2}$-like products formed in enamel by the treatment with FD [Tenuta and Cury, 2013].

In summary, our findings confirmed that fluorotic enamel is more susceptible to demineralization than sound enamel, but we extended this knowledge showing that the use of fluoride dentifrice overcame this deficiency. The combination of the topical effect of fluoride from community fluoridation programs and FD use should be object of further studies.

\section{ACKNOWLEDGMENTS}

The manuscript was based on the first author's Master thesis for the Graduate Program 
in Dentistry, Cariology area, Piracicaba Dental School, University of Campinas, Brazil. The first author received a scholarship during her Master in Dentistry from FAPESP (grant $n^{\circ} 2016 / 24190-1$ ), Brazil. This manuscript is an outcome of a research project that also is supported by CNPq (grant $n^{\circ} 307270 / 2015-7$ ), Brazil.

\section{AUTHOR CONTRIBUTIONS}

Conceived and designed the experiments: JAC; performed the experiment: LFA; analyzed the data: LFA, LMM, JAC, EAMM; wrote the paper: LFA, JAC; revised the paper: LMM, EAMM.

\section{REFERENCES}

Alhawij H, Lippert F, Martinez-Mier EA. Relative fluoride response of caries lesions created in fluorotic and sound teeth studied under remineralizing conditions. J Dent. 2015 Jan;43(1):103-9.

Argenta RM, Tabchoury CP, Cury JA. A modified pH-cycling model to evaluate fluoride effect on enamel demineralization. Pesqui Odontol Bras. 2003 Jul-Sep; 17(3):241-6.

Arnold FA, Likins RC, Russell AL, Scott DB. 1962. Fifteenth year of the Grand Rapids fluoridation study. The Journal of the American Dental Association, Volume 65, Issue 6, $780-785$.

Baelum V, Manji F, Fejerskov O. Posteruptive tooth age and severity of dental fluorosis in Kenya. Scand J Dent Res. 1986 Oct; 94(5):405-10.

Burt BA. 1992. The changing patterns of systemic fluoride intake. J Dent Res. 71(5):1228-1237.

Chankanka O, Levy SM, Warren JJ, Chalmers JM: A literature review of aesthetic perceptions of dental fluorosis and relationships with psychosocial aspects/oral healthrelated quality of life. Community Dent Oral Epidemiol 2010; 38: 97-109.

Chen S, Eisenmann DR. (1984). Ultrastructural study of the effects of fixation and fluoride injection on stippled material during amelogenesis in the rat. Arch Oral Biol 29:681-686.

Cury JA, de Oliveira BH, dos Santos AP, Tenuta LM. Are fluoride releasing dental materials clinically effective on caries control? Dent Mater. 2016 Mar; 32(3):323-33. 
Cury JA, do Amaral RC, Tenuta LM, Del Bel Cury AA, Tabchoury CP: Low-fluoride toothpaste and deciduous enamel demineralization under biofilm accumulation and sucrose exposure. Eur J Oral Sci 2010; 118: 370-375.

Cury JA, Rebelo MA, Del Bel Cury AA, Derbyshire MT, Tabchoury CP. Biochemical composition and cariogenicity of dental plaque formed in the presence of sucrose or glucose and fructose. Caries Res. 2000 Nov-Dec; 34(6):491-7.

Cury JA, Tenuta LM. Enamel remineralization: controlling the caries disease or treating early caries lesions? Braz Oral Res. 2009; 23 Suppl 1:23-30.

Do LG. Distribution of caries in children: variations between and within populations. J Dent Res. 2012 Jun; 91(6):536-43.

Featherstone JD, ten Cate JM, Shariati M, Arends J. Comparison of artificial caries-like lesions by quantitative microradiography and microhardness profiles. Caries Res. 1983; 17(5):385-91.

Fejerskov O, Larsen MJ. 2015. Demineralization and remineralization: the key to understanding clinical manifestations of dental caries. Fejerskov O, Nyvad B, Kidd E, editors. In Dental Caries: The Disease and Its Clinical Management. Third Edition ed. Oxford: Wiley-Blackwell. pp. 155-170.

Fejerskov O, Silverstone LM, Melsen B, Moller IJ. Histological features of fluorosed human dental enamel. Caries Res. 1975; 9(3):190-210.

Fejerskov O, Thylstrup A, Larsen MJ. Rational use of fluorides in caries prevention. A concept based on possible cariostatic mechanisms. Acta Odontol Scand. 1981; $39(4): 241-50$.

Holmen L, Thylstrup A, Årtun J: Clinical and Histological Features Observed during Arrestment of Active Enamel Carious Lesions in vivo. Caries Res 1987; 21:546-554.

Kidd EAM, Thylstrup A, Fejerskov O, Bruun C: Influence of fluoride in surface enamel and degree of dental fluorosis on caries development in vitro. Caries Res 1980; 14: 196202.

Kidd EA, Thylstrup A, Fejerskov O, Silverstone LM. Histopathology of caries-like lesions created in vitro in fluorosed and sound enamel. Caries Res. 1978; 12(5):268-74. 
Kielbassa AM, Wrbas KT, Schulte-Mönting J, Hellwig E. Correlation of transversal microradiography and microhardness on in situ-induced demineralization in irradiated and nonirradiated human dental enamel. Arch Oral Biol. 1999 Mar;44(3):243-51.

Lima CV, Tenuta LMA, Cury JA. Fluoride Increase in Saliva and Dental Biofilm due to a Meal Prepared with Fluoridated Water or Salt: A Crossover Clinical Study. Caries Res. 2018 Jun $7 ;$ 53(1):41-48.

Lippert F, Butler A, Lynch RJ, Hara AT. Effect of fluoride, lesion baseline severity and mineral distribution on lesion progression. Caries Res. 2012; 46(1):23-30.

Marín LM, Cury JA, Tenuta LM, Castellanos JE, Martignon S. Higher Fluorosis Severity Makes Enamel Less Resistant to Demineralization. Caries Res. 2016; 50(4):407-13.

Marinho VC, Higgins JP, Logan S, Sheiham A. Fluoride toothpastes for preventing dental caries in children and adolescents. Cochrane Database Syst. Rev In: The Cochrane Library, Issue 1, 2003.

Moreno EC, Kresak M, Zahradnik RT. Fluoridated hydroxyapatite solubility and caries formation. Nature 1974; $247:$ 64-5.

Moreno EC, Zahradnik RT. Chemistry of enamel subsurface demineralization in vitro. J Dent Res. 1974 Mar-Apr; 53(2):226-35.

Mullen J, McGaffin J, Farvardin N, Brightman S, Haire C, Freeman R. Caries status in 16 year-olds with varying exposure to water fluoridation in Ireland. Community Dent Health. 2012 Dec; 29(4):293-6.

Nelson DGA. The influence of carbonate on the atomic structure and reactivity of hydroxyapatite. J Dent Res 1981; 60: 1621-9.

Nobre dos Santos M, Cury JA. Dental plaque fluoride is lower after discontinuation of water fluoridation. Caries Res. 1988; 22(5):316-7.

Petersen PE, Bourgeois D, Bratthall D, Ogawa H. Oral health information systems-towards measuring progress in oral health promotion and disease prevention. Bull World Health Organ. 2005 Sep; 83(9):686-93. Epub 2005 Sep 30.

Petersen PE, Lennon MA: Effective use of fluorides for the prevention of dental caries in the 21st century: the WHO approach. Community Dent Oral Epidemiol 2004; 32: 319321. 
Richards A, Fejerskov O, Baelum V. Enamel fluoride in relation to severity of human dental fluorosis. Adv Dent Res. 1989 Sep;3(2):147-53.

Riordan PJ. Perceptions of dental fluorosis. J Dent Res. 1993 Sep; 72(9):1268-74.

Russell AL, Hamilton PM. Dental caries in permanent first molars after eight years of fluoridation. Archs Oral Biol (Spec Suppl) 1961; 6:50-7.

Slade GD, Grider WB, Maas WR, Sanders AE. Water Fluoridation and Dental Caries in U.S. Children and Adolescents. J Dent Res. 2018 Sep; 97(10):1122-1128.

Spencer AJ, Do LG, Ha DH. Contemporary evidence on the effectiveness of water fluoridation in the prevention of childhood caries. Community Dent Oral Epidemiol. 2018 Aug; 46(4):407-415.

Spencer AJ, Do LG, Mueller U, Baines J, Foley M, Peres MA. Understanding Optimum Fluoride Intake from Population-Level Evidence. Adv Dent Res. 2018 Mar; 29(2):144156.

Suma R, Shashibhushan KK, Shashikiran ND, Subba RV. Progression of artificial caries in fluorotic and nonfluorotic enamel: an in vitro study. J Clin Pediatr Dent. 2008 Winter; 33(2):127-30.

ten Cate JM. Current concepts on the theories of the mechanism of action of fluoride. Acta Odontol Scand. 1999; 57(6):325-9.

Thylstrup A, Fejerskov O. Clinical appearance of dental fluorosis in permanent teeth in relation to histologic changes. Community Dent Oral Epidemiol. 1978 Nov; 6(6):315-28. Waidyasekera PG, Nikaido T, Weerasinghe DD, Wettasinghe KA, Tagami J. Caries susceptibility of human fluorosed enamel and dentine. J Dent. 2007 Apr; 35(4):343-9.

Yamazaki H, Litman A, Margolis HC. Effect of fluoride on artificial caries lesion progression and repair in human enamel: regulation of mineral deposition and dissolution under in vivo-like conditions. Arch Oral Biol. 2007 Feb; 52(2):110-20. Epub 2006 Oct 16.

Figure 4. Concentration of fluoride in enamel before (baseline) and after the $\mathrm{pH}$-cycling regimen and treatments with dentifrices PD (Fig.4a) or FD (Fig.4b), and according to the distance of enamel surface $(\mu \mathrm{m})$. 
Figure 5. Mean and SD of fluoride formed in enamel $(\mu \mathrm{g} \mathrm{F} / \mathrm{g})$ by the treatments with PD or FD dentifrices according to the TF scores. Distinct capital letters show differences statistically significant $(p<0.05)$ among the TF groups within treatment with PD or FD. 
Figure 1. Flow chart of the experimental design

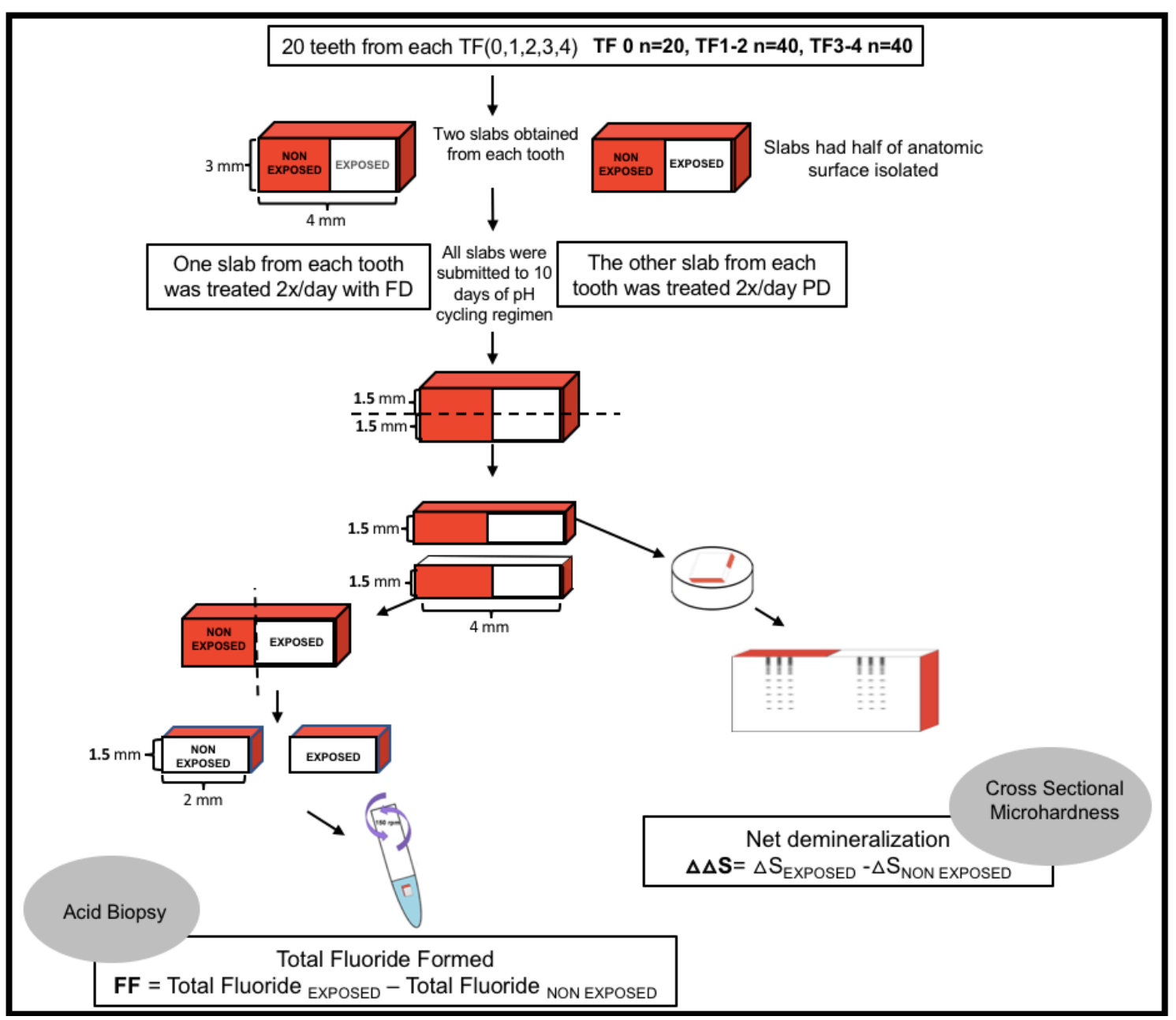


Table 1. Two-way ANOVA ( $p$ values) of the data

\begin{tabular}{|c|c|c|c|}
\hline \multirow[b]{2}{*}{ Variables } & \multicolumn{2}{|c|}{ Factors } & \multirow{2}{*}{$\begin{array}{c}\text { Interaction } \\
\text { (TF * Dentifrices) }\end{array}$} \\
\hline & $\begin{array}{c}\text { Fluorosis } \\
\text { (TF) }\end{array}$ & Dentifrices & \\
\hline Demineralization $(\Delta \Delta S)$ & 0.002 & $<0.001$ & $<0.0001$ \\
\hline Fluoride formed (FF) & $<0.001$ & $<0.001$ & 0.008 \\
\hline
\end{tabular}



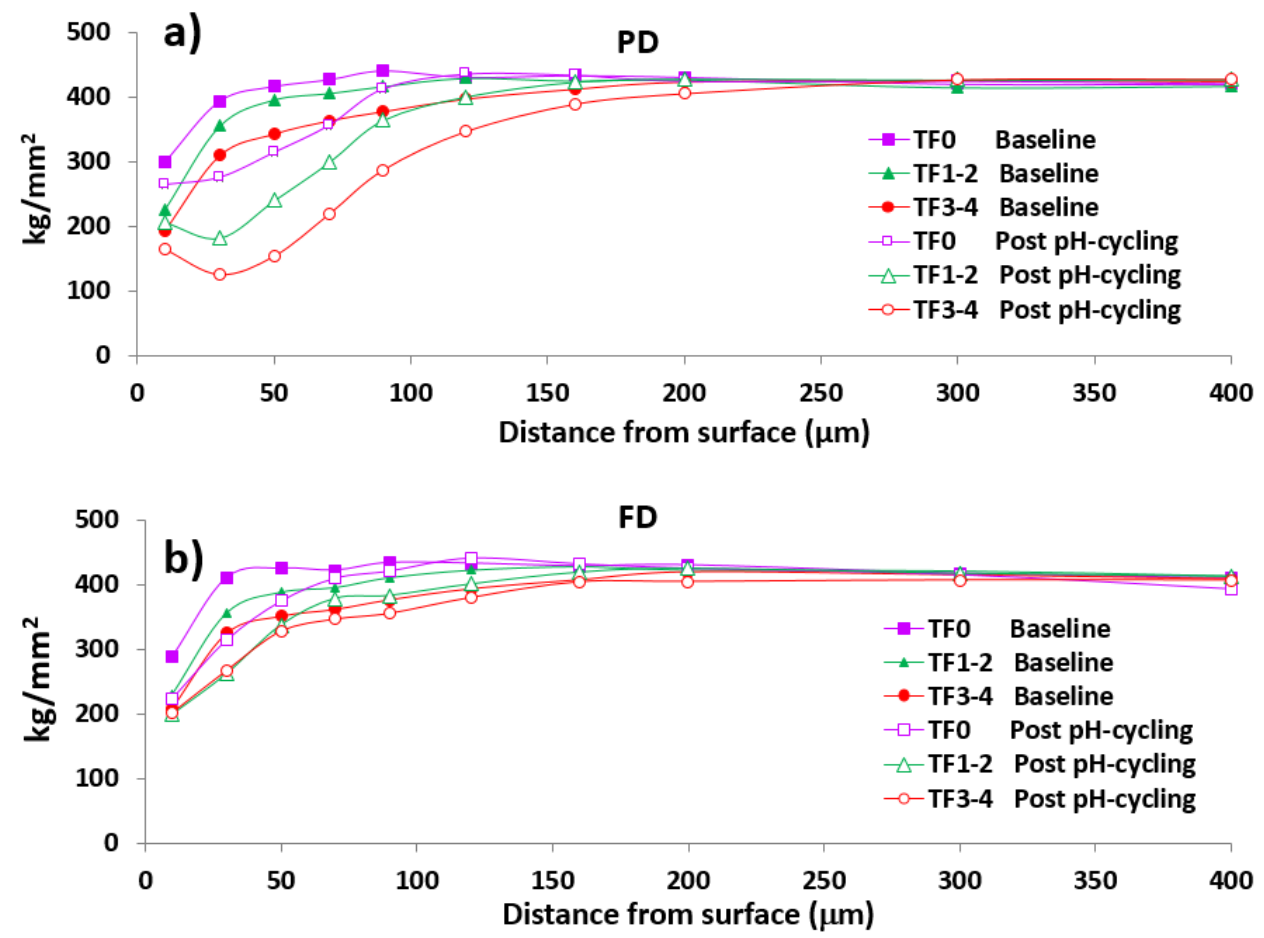

Figure 2. Hardness $\left(\mathrm{kg} / \mathrm{mm}^{2}\right)$ profile of enamel before (baseline) and after the $\mathrm{pH}$-cycling regimen and treatment with PD (Fig. 2a) or FD (Fig. 2b), according to distance ( $\mu \mathrm{m}$ ) from the surface and TF score. 


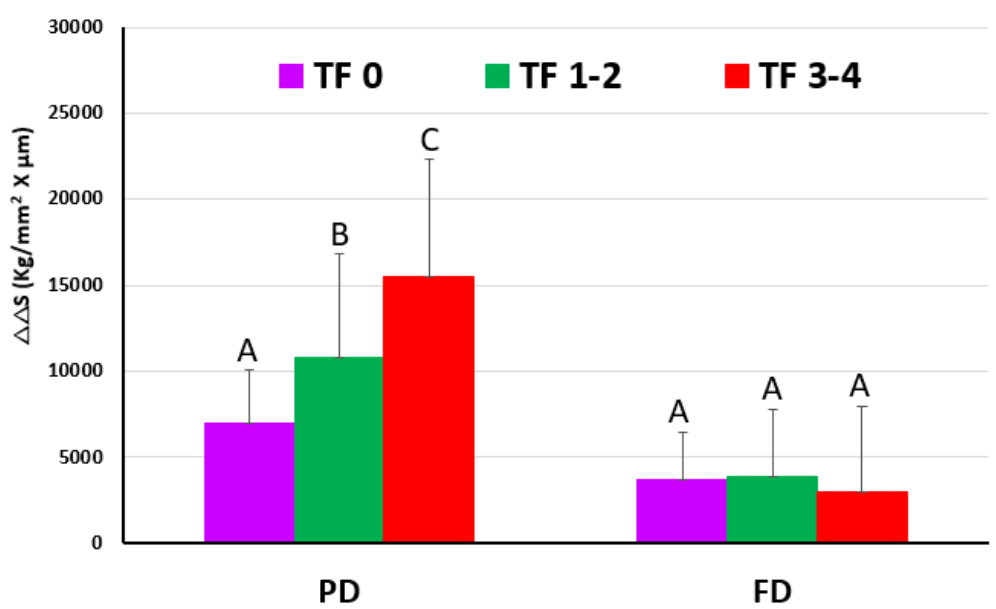

Figure 3. Mean and SD of net area of demineralization $(\Delta \Delta S)$ found, according to the TF scores and treatment groups with PD or FD. Distinct letters show differences statistically significant $(p<0.05)$ among the TF scores within the groups of dentifrice treatments, PD and FD. 
PD

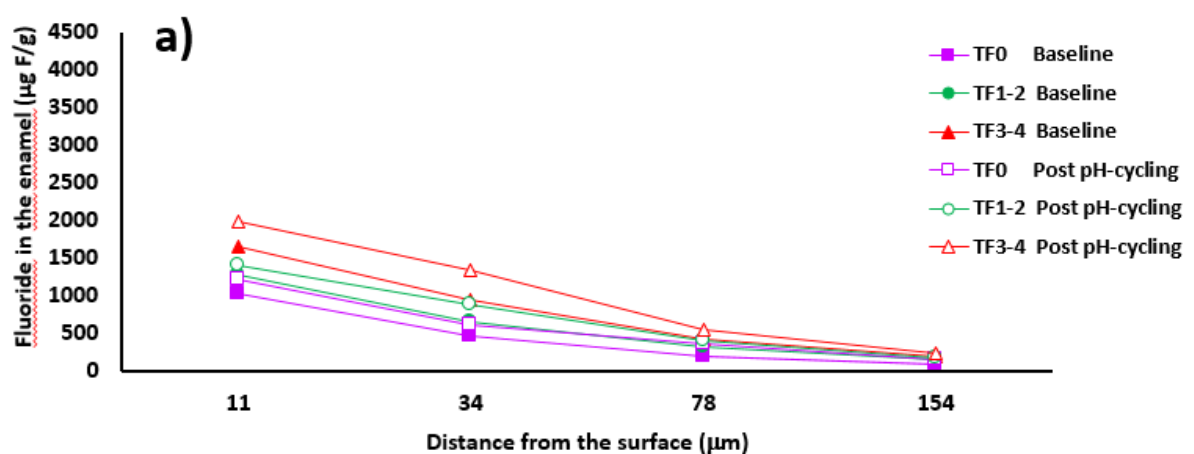

FD

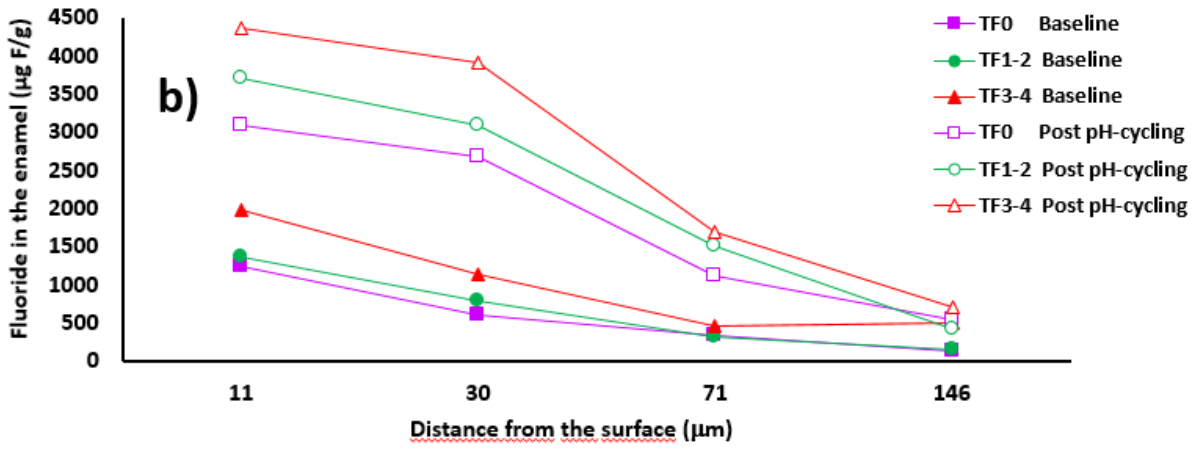

Figure 4. Concentration of fluoride in enamel before (baseline) and after the pH-cycling regimen and treatments with dentifrices PD (Fig.4a) or FD (Fig.4b), and according to the distance of enamel surface $(\mu \mathrm{m})$. 


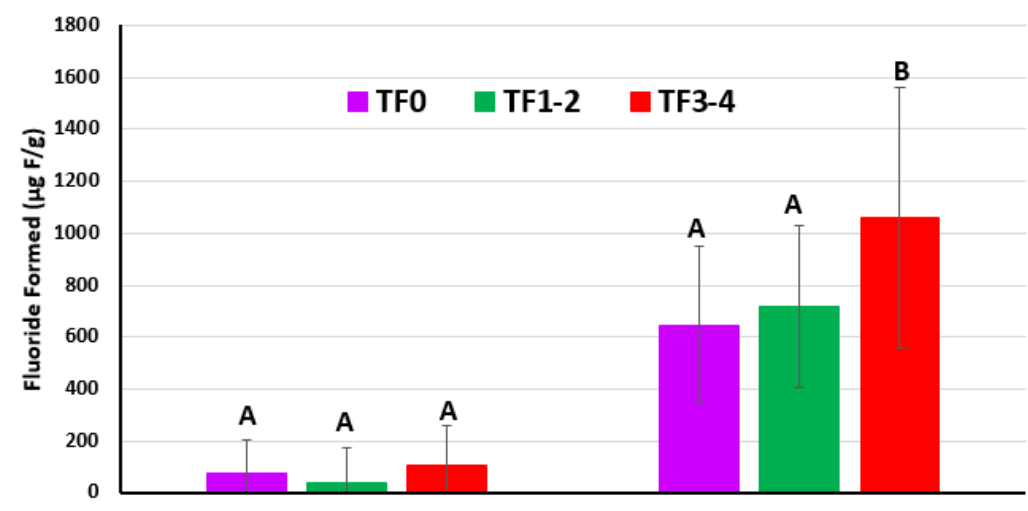

PD

FD

Figure 5. Mean and SD of fluoride formed in enamel ( $\mu \mathrm{g} \mathrm{F/g}$ ) by the treatments with PD or FD dentifrices according to the TF scores. Distinct capital letters show differences statistically significant $(p<0.05)$ among the TF groups within treatment with PD or FD. 